\title{
Low-cost resonant cavity Raman gas probe for multi-gas detection
}

\author{
J. Thorstensen \\ jostein.thorstensen@sintef.no \\ K. H. Haugholt
}

\section{A. Ferber}

\section{K. A. H. Bakke}

\section{J. Tschudi}

\author{
SINTEF ICT, Optical measurement systems, 0373 Oslo, Norway \\ SINTEF ICT, Optical measurement systems, 0373 Oslo, Norway \\ SINTEF ICT, Optical measurement systems, 0373 Oslo, Norway \\ SINTEF ICT, Optical measurement systems, 0373 Oslo, Norway
}

Raman based gas sensing can be attractive in several industrial applications, due to its multi-gas sensing capabilities and its ability to detect $\mathrm{O}_{2}$ and $\mathrm{N}_{2}$. In this article, we have built a Raman gas probe, based on low-cost components, which has shown an estimated detection limit of $0.5 \%$ for 30 second measurements of $\mathrm{N}_{2}$ and $\mathrm{O}_{2}$. While this detection limit is higher than that of commercially available equipment, our estimated component cost is approximately one tenth of the price of commercially available equipment. The use of a resonant Fabry-Pérot cavity increases the scattered signal, and hence the sensitivity, by a factor of 50 . The cavity is kept in resonance using a piezo-actuated mirror and a photodiode in a feedback loop.

The system described in this article was made with minimum-cost components to demonstrate the low-cost principle. However, it is possible to decrease the detection limit using a higher-powered (but still low-cost) laser and improving the collection optics. By applying these improvements, the detection limit and estimated measurement precision will be sufficient for e.g. the monitoring of input gases in combustion processes, such as e.g. (bio-)gas power plants. In these processes, knowledge about gas compositions with $0.1 \%$ (absolute) precision can help regulate and optimize process conditions.

The system has the potential to provide a low-cost, industrial Raman sensor that is optimized for specific gas-detection applications.

[DOI: http://dx.doi.org/10.2971/jeos.2014.14054]

Keywords: Raman scattering, gas spectroscopy, Fabry-Pérot cavity

\section{INTRODUCTION}

Gas sensors find their use in many applications, ranging from health and safety, to process control and environmental monitoring. Several detection technologies exist, including electrochemical, semiconductor and infrared sensors. Electrochemical and semiconductor gas sensors are reasonably low-cost, but suffer from cross-sensitivity, which can be mitigated to some extent using sensor arrays [1]-[3] and limited lifetime or short calibration intervals. These sensors inherently provide no or limited information about multi-gas mixtures.

Optical spectroscopy is a valuable tool when it comes to the identification of gases and gas compositions. Infrared spectroscopy is the most commonly used, where the absorption or emission of specific wavelengths from a gas is measured. Spectroscopic gas sensors can be highly specific (i.e. low crosssensitivity) and e.g. Fourier Transform Infrared (FTIR) spectrometers can give quantitative information about complex gas mixtures [4]. Single gas detectors can be quite compact and low-cost $[5,6]$.

Raman spectroscopy relies on the inelastic scattering of pho- tons by molecules in the gas, whereby the energy shift of the photon identifies the scattering gas. Raman active transitions are, in general, different than IR active transitions [7], e.g. $\mathrm{O}_{2}$, $\mathrm{N}_{2}, \mathrm{H}_{2}$ and $\mathrm{Cl}_{2}$ (all diatomic homonuclear gases) are invisible in IR spectroscopy, while they are visible in Raman spectroscopy. The ability to measure $\mathrm{O}_{2}, \mathrm{~N}_{2}$ and $\mathrm{H}_{2}$ along with the multi-gas sensing capabilities of optical spectroscopy are desirable in several applications, for example fuel gas sensing for natural- or biogas power plants, where $\mathrm{CH}_{4}, \mathrm{CO}_{2}, \mathrm{O}_{2}$, $\mathrm{N}_{2}$ and $\mathrm{H}_{2}$ are relevant to monitor [8]. Other processes where $\mathrm{CO}, \mathrm{NO}_{x}, \mathrm{~N}_{2}, \mathrm{O}_{2}$ need quantification or where the infrared spectrum is dominated by $\mathrm{H}_{2} \mathrm{O}$ absorption can also be relevant for Raman gas sensors since they are very weakly affected by water [9]. Further advantages of Raman gas sensors are that they can tolerate high gas concentrations and have a high dynamic range since they do not suffer from saturation effects [10]. The measurement volume can be very small in Raman spectroscopy [11], allowing for miniaturized measurement cells or measurements in e.g. micro-reactors for e.g. combinatorial chemistry. 
The main drawback of Raman spectroscopy is that the Raman scattering process is fairly weak, meaning that it is difficult to obtain acceptable sensitivity. As such, the majority of Raman based spectrometers are built for sensing in solids or liquids, where the higher material density increases the signal significantly. For these applications, hand-held Raman spectrometers are available [12]-[14]. For sensing of one specific chemical compound, Surface-Enhanced Raman Spectroscopy (SERS) can give greatly increased sensitivity for species adsorbed to a nano-structured surface [15].

For Raman gas sensing, a multitude of sensing techniques has been developed in order to overcome the challenge of weak signal strength. Non-linear techniques such as Photoacoustic Raman spectroscopy, Coherent Anti-stokes Raman spectroscopy and Stimulated Gain Raman Spectroscopy [16], nonresonant multi-pass cavities [17]-[21], capillary cells or hollow photonic crystal fibers [22, 23], high-pressure cells [9, 24], resonant Fabry-Pérot cavities (FP-cavities) using directly coupled cavities [25], optical feedback [26] or intra-cavity designs [27] have all been demonstrated. While all of these approaches increase the probe laser intensity, their increased complexity leads to fairly high-cost Raman gas sensors.

The purpose of this paper is to describe a gas probe that combines the capabilities of multi-gas sensing through optical spectroscopy with the Raman capabilities of $\mathrm{O}_{2}, \mathrm{~N}_{2}$ and $\mathrm{H}_{2}$ detection in a low-cost design. It is our belief that a significant reduction in price for Raman systems would enable entry into new application areas in gas detection. This paper describes a low-cost Raman gas probe that overcomes the problem of weak signal by using a resonant Fabry-Pérot cavity. High-cost components such as high-power lasers and highcost spectrometers are replaced with a simple, low-cost laser pointer and a low-cost spectrometer. Even filter-based point sensors for specific gases can be envisioned, reducing the costs even further. The set-up, which comprises standard optical components, is used to demonstrate the potential for a lowcost Raman system for gas detection. The detection limit for the set-up is estimated at $0.5 \%$ for 30 second measurements of $\mathrm{N}_{2}$ and $\mathrm{O}_{2}$.

\section{THEORY OF FP-CAVITIES}

Rearranging equations in [28], the theoretical gain of the cavity in resonance, $G$, is given by the expression

$$
G_{\text {cavity }} \simeq \frac{4 T_{1}}{\left(T_{1}+T_{2}+A\right)^{2}}
$$

Where $T_{1}$ and $T_{2}$ are the transmittivities of the in- and outcoupling mirrors, and $A$ is the round-trip loss. This expression is maximized for $T_{1}=T_{2}+A$, i.e. the incoupling matches the round-trip losses including out-coupling, and $T_{2}$ and $A$ as small as possible. The finesse of the cavity is found by monitoring the cavity transmission as the length of the cavity is swept through one free spectral range. The round-trip loss, $A$, can then be estimated through the expression

$$
F \simeq \frac{2 \pi}{T_{1}+T_{2}+A}
$$

where the transmittivities can be measured. The total gain, $G_{t o t}$, was estimated by measuring the transmitted power from the cavity, $P_{\text {trans }}$ by using the relation

$$
G_{\text {tot }} \stackrel{\text { def }}{=} \frac{P_{\text {cavity }}}{P_{\text {laser }}}=\frac{P_{\text {trans }}}{P_{\text {laser }} T_{2}}=G_{\text {cavity }} \eta_{\text {coupl }}
$$

Where $P_{\text {laser }}$ is the power of the probe laser, and $P_{\text {cavity }}$ is the power circulating in the cavity. $\eta_{\text {coupl }}$ is the ratio between the measured gain and the gain expected from Eq. (1).

\section{EXPERIMENTAL SET-UP}

The experimental setup is shown in Figure 1. A simple frequency-doubled YAG laser pointer with $50 \mathrm{~mW}$ output power was used as a probe laser. The mode field of the laser was matched to that of the cavity using a focusing lens. The Fabry-Pérot cavity comprises two highly reflecting mirrors. One of the cavity mirrors is mounted on piezo-actuators. A photodiode measures the cavity transmission, and a microcontroller-based feedback loop seeks to maximize transmission and thereby the gain in the cavity. The photodiode is placed at the output of the cavity. The $\lambda / 4$ plate and the beam splitter serve as an optical isolator, blocking the specularly reflected light from re-entering the probe laser. The readout of the Raman signal was done by placing an optical fiber directly adjacent to the laser beam in the cavity. A fiber coupled spectrometer that rejected the probe wavelength recorded the Raman signal. The spectrometer is an OceanOptics USB2000+ spectrometer with a silicon detector, a $50 \mu \mathrm{m}$ wide input slit and a spectral resolution of $1.4 \mathrm{~nm}$.

The electronic feedback-loop for the cavity length adjustment works as follows:

- Firstly, a complete (approx. $10 \mathrm{~V}$ ) voltage scan is performed. The voltage with the maximum transmitted intensity is recorded.

- After the complete scan, subsequent scans are performed in a smaller voltage range around a transmission peak.

- Finally, the feedback-loop is activated, wherein the voltage is increased or decreased in small steps, moving in the direction of higher transmitted light intensity.

\section{RESULTS}

An outcoupling mirror with transmission of $0.04 \%$ and an incoupling mirror transmission of $1 \%$ were used. Sweeping the voltage over the piezo actuator (Figure 2), $F$ and $G_{t o t}$ can be measured from the width (FWHM) and height of the peak and the distance between the peaks (free spectral range, FSR). Using Eq. (2) and a measured finesse of approx. 400, we estimate $\mathrm{A}=6 \mathrm{E}-3$, i.e. a scattering loss of $3 \mathrm{E}-3$ at each mirror assuming equal loss in the two mirrors. Using this value for $\mathrm{A}$ in Eq. (1) it is expected that $G_{\text {cavity }}=150$. Measurements show $G_{t o t}=50$, and hence, a coupling efficiency of around $1 / 3$ is estimated. Reasons for this loss could be mismatch between the laser and cavity modes due to differences in position of beam 


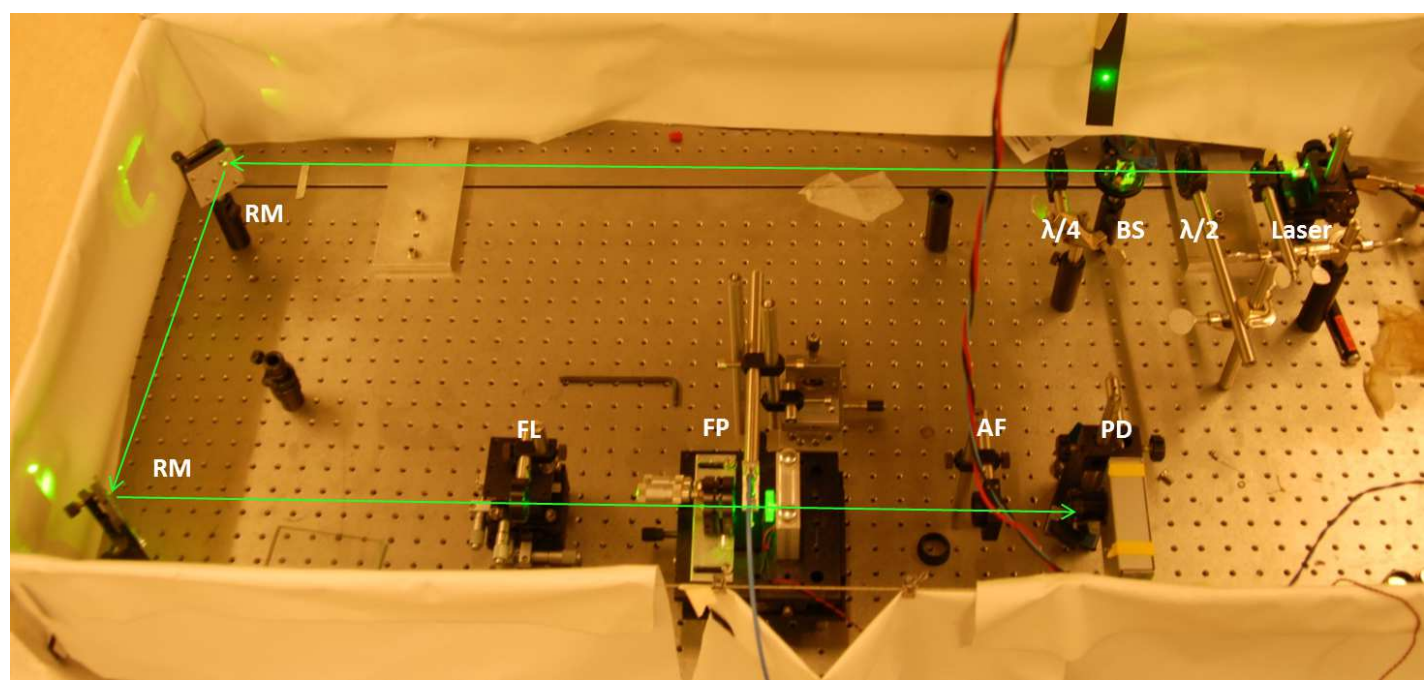

FIG. 1 The experimental setup for the Raman gas probe, with the components marked. Laser: source laser and IR filter, $\lambda / 2$ : half wave plate, BS: polarizing beam splitter, $\lambda / 4$ : quarter wave plate, RM: relay mirrors, FL: focusing lens, FP: Fabry-Pérot cavity with detector fiber, AF: attenuation filter, PD: photodiode.

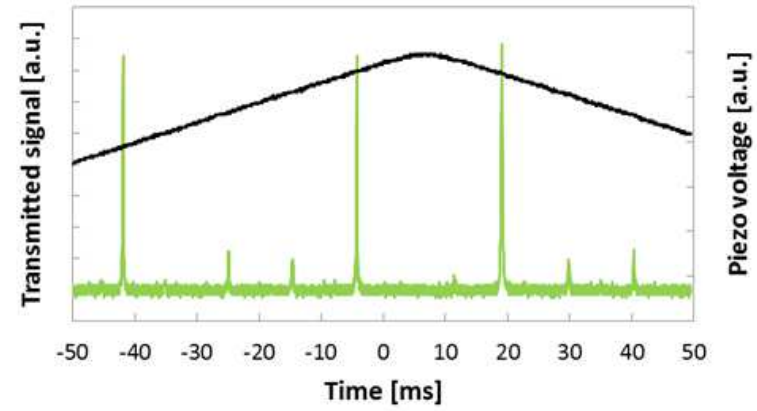

FIC. 2 Measurement of the cavity transmission (green, left $y$-axis) as the voltage over the piezo actuator (black, right $y$-axis) is swept. Small transmission peaks between the main transmission peaks are seen, corresponding to non-gaussian resonator modes.

waist, waist diameter or beam angle [29]. With $50 \mathrm{~mW}$ input power, this means that there is $2.5 \mathrm{~W}$ of optical power circulating in the cavity. As the Raman signal is directly proportional to the optical power, the Raman signal is also increased by a factor of 50 .

Currently, the control algorithm for cavity length adjustment loses track of the transmission peak from time to time. The reason for this is still unknown, but may be related to unstable laser modes or hysteresis in the piezo actuators. It is estimated that the time-averaged transmission is around half of the peak transmission seen in Figure 2.

As a gas cell has yet to be constructed, measurements have only been performed on lab air. The results are shown in Figure 3. Two Raman spectra are shown in black and green, and the expected position of the Stokes lines of nitrogen and oxygen are marked in red and blue respectively. The black spectrum is obtained in 30 seconds. The green spectrum is obtained in only 4 seconds, indicating the potential for fairly quick measurements with lower precision. In order to properly calculate the precision and accuracy of the gas probe, calibration should be performed in a gas cell using several different known gas concentrations. Such a gas cell has not been available for this work; however, we can use the signal to

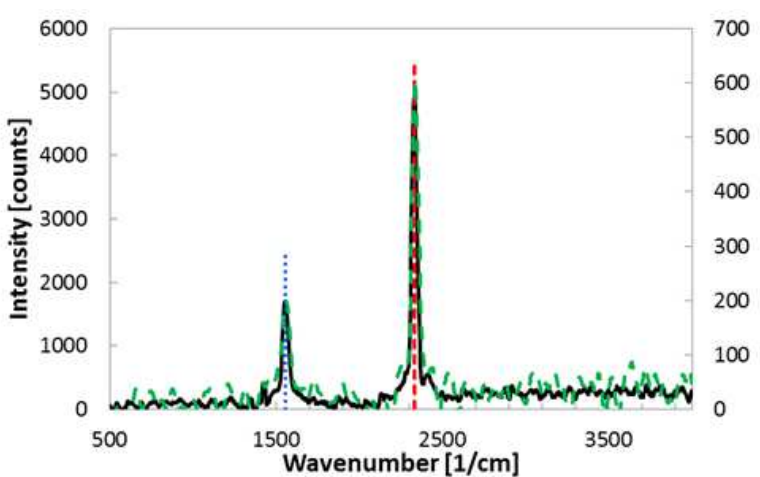

FIG. 3 Raman spectra of $\mathrm{O}_{2}$ and $\mathrm{N}_{2} .30 \mathrm{sec}$ (solid black, left y-axis) and $4 \mathrm{sec}$ (dashed green, right $\mathrm{y}$-axis) measurement time are shown. The expected position of $\mathrm{O}_{2}$ (1555 1/cm, dotted blue) and $N_{2}(23301 / \mathrm{cm}$, dashed red) Stokes lines are also shown.

noise ratio and the atmospheric concentration of $\mathrm{O}_{2}$ and $\mathrm{N}_{2}$ in order to estimate a detection limit of around $0.5 \%$ for $\mathrm{O}_{2}$ and $\mathrm{N}_{2}$. As the strength of the Raman scattered signal is proportional to the Raman scattering cross-section, the detection limits of different gases will be different than the detection limits of $\mathrm{O}_{2}$ and $\mathrm{N}_{2}$. A non-exhaustive list of Raman scattering crosssections is given in [9], indicating that the Raman scattering cross-section can be a factor 10 higher or lower than that of $\mathrm{N}_{2}$ and $\mathrm{O}_{2}$ (having similar Raman scattering cross-sections). One commercially-available, high-cost Raman gas probe is able to detect $\mathrm{N}_{2}$ at ambient pressure with concentration on the order of 50 ppm with 1 second measurement time, i.e. a factor 100 lower detection limit.

\section{DISCUSSION}

While the current setup has demonstrated the ability to measure $\mathrm{O}_{2}$ and $\mathrm{N}_{2}$ with an estimated detection limit of $0.5 \%$ for 30 seconds measurement time, significant improvements of the current detection limit can be obtained primarily by increasing light coupling to the spectrometer and by increasing the laser power. Laser pointers with $500 \mathrm{~mW}$ power (i.e. 
a power increase of a factor 10) are readily available at a cost of 500 USD and less (e.g. the LZCR532-500 from Lazerer at 299 USD). Using a fiber bundle to collect the Raman scattered light and to deliver that light through the input slit of the spectrometer would realistically improve light collection with a factor 10 . These improvements would reduce the detection limit by a factor of 100 while keeping the system costs down. Such an improvement would bring the detection limit of this system down towards that of commercially available high-cost Raman gas sensors. A $500 \mathrm{~mW}$ laser pointer and a gain factor of 50 would result in a circulating power of $25 \mathrm{~W}$ in the FP-cavity. Reaching the same Raman scattered signal without the FP-cavity would require a $25 \mathrm{~W}$ laser. Lasers of such high power are still very costly. As such, the FP-cavity provides a means of using low-cost, low-power laser sources.

The current setup demonstrates the potential for a low-cost Raman spectrometer. In [15], a cost estimate for a Raman probe for liquids is given, where the laser and high cost spectrometers are emphasized as the main cost drivers. It is argued that with the use of a low-cost laser and spectrometer, the cost of a Raman system may be reduced from around 100000 USD to below 4000 USD. In addition to the components described in [15], the gas probe described in this work requires an actively adjusted gas cell with a feedback loop because of its application in gas-sensing. The estimated total component cost of this system is still below 12500 USD.

The absolute precision of a spectrometer is given by a sum of the readout noise of the spectrometer and the shot-noise (photon noise) arising from the photon statistics. As such, the absolute precision will decrease, while the relative precision will increase with increasing gas concentration. In the configuration described in this article, the readout noise dominates for low gas concentrations, and amounts to around $0.5 \%$ (absolute). This is stated as the detection limit, for a signal to noise ratio of 1 . For $100 \%$ gas concentration, the noise is dominated by shot-noise, and is estimated to around $1.2 \%$ (absolute). With a factor 100 stronger signal as outlined in the beginning of this chapter, the detection limit will decrease by a factor 100 to around $50 \mathrm{ppm}$ (absolute), while the precision at $100 \%$ gas concentration will increase by a factor 10 (as the shot-noise equals the square root of the signal), giving an uncertainty of around $0.1 \%$ (absolute). Other gases will have different Raman scattering cross-sections, and the noise will scale accordingly.

The overall probe laser gain, $G_{t o t}$, was estimated to around 50. As such, our system provides a gain factor similar to that obtained in non-resonant multi-pass cavities [17]-[21]. Significantly higher $G_{\text {cavity }}$ can be obtained using super-polished mirrors with high reflectivity. However, such mirrors would soon become contaminated in a real measurement situation, rapidly deteriorating $G_{\text {cavity }}$. As such, the extra cost of such mirrors cannot be justified in this application. The optical feedback is sensitive to vibrations and frequency shifts in the laser. The stability of this feedback could be improved e.g. by applying mechanically biased piezo-actuators in order to improve response time and decrease hysteresis in the piezoactuators. Fluorescence can be a problem when doing liquid and solid state Raman analysis, as the fluorescence signal can

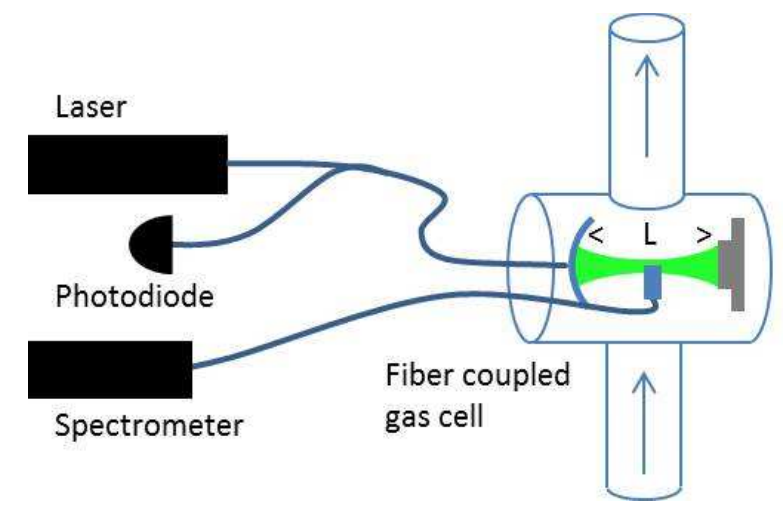

FIG. 4 Sketch of prototype Raman gas probe. The laser and feedback loop photodiode are both fiber coupled. The cavity end mirror has been replaced by a piezoelectric silicon micro mirror, and the probe light is delivered to the gas cell by an optical fiber. The length of the cavity, L, can be as small as a couple of millimeters, depending on the application.

be much stronger than the Raman signal. For gas-phase Raman analysis, only very few compounds, such as ketones, fluoresce. These are normally excited using UV wavelengths [30], and are as such not expected to be an issue with the current $532 \mathrm{~nm}$ excitation wavelength.

An industrial prototype of this gas probe could be envisioned as shown in Figure 4 . The use of a piezo-actuated micro mirror $[31,32]$ as the cavity end mirror could enable miniaturization of the cavity. In the figure, the length of the cavity, L, can be as small as a couple of millimeters or as large as several centimeters, depending on the application. Fiber coupling of all components increase robustness and simplifies design. The FP-cavity is drawn inside of the gas cell. As seen from Eq. (1), any cavity loss would reduce $G_{\text {cavity. }}$. The windows of a gas cell would introduce such losses. Preliminary experiments inserting anti-reflection coated glass windows into the cavity reduced $G_{\text {cavity }}$ by a factor of two. As such, it is more desirable to put the FP-cavity inside of the gas cell, or to use the end mirrors of the FP-cavity as the windows to the gas cell.

Concrete applications for this gas-probe would be processes where \%-range concentrations of multiple gases should be measured in real-time. In combustion processes, gas composition monitoring is important, both for emission control, and for process regulation and optimization. As such, the monitoring of the main fuel gas species $\mathrm{CH}_{4}, \mathrm{CO}_{2}, \mathrm{O}_{2}, \mathrm{~N}_{2}$ and $\mathrm{H}_{2}$ or flue gas species $\mathrm{CO}_{2}, \mathrm{H}_{2} \mathrm{O}, \mathrm{O}_{2}$ and $\mathrm{N}_{2}$ is relevant. Relevant detection limits in this process could be on the order of $0.1 \%$ $[33,34]$. New "green" technologies can be demanding in terms of varying gas concentrations in the input gas. Some examples are: biogas power plants, where the composition of the bio-gas can be varying over time [35] and oxy-fuel combustion power plants for carbon capture and sequestration (CCS) applications, where combustion air is replaced by oxygen and recirculation of part of the flue gas must be real-time controlled [36]. In both of these processes, real-time monitoring of the composition of the input gases can help optimizing the combustion process. Again, detection limits in the $0.1 \%$ range would be suitable. The Raman gas probe described herein, combined with a higher power low-cost laser, would provide suitable precision for such applications. 


\section{CONCLUSIONS}

A Raman gas probe, based on low-cost components, has been successfully demonstrated. Our current detection limit is estimated to $0.5 \%$ for 30 second measurements of $\mathrm{N}_{2}$ and $\mathrm{O}_{2}$.

While the achieved detection limit is higher than that of commercially available equipment, the use of higher powered low-cost lasers, along with improved collection of the Raman scattered signal would reduce the detection limit towards that of commercially available equipment, while still keeping component costs at around a tenth of the price of commercially available equipment. Such a system would open up for the possibility of low-cost Raman gas-sensing. A relevant application segment for the Raman gas probe could be combustion processes, where detection precision on the order of $0.1 \%$ (absolute) in multi-gas measurements would be relevant for process monitoring and control.

\section{References}

[1] C. C. Austin, B. Roberge, and N. Goyer, "Cross-sensitivities of electrochemical detectors used to monitor worker exposures to airborne contaminants: false positive responses in the absence of target analytes," J. Environ. Monitor. 8(1), 161-166 (2006).

[2] H. Meixner, and U. Lampe, "Metal oxide sensors," Sensor. Actuat. B-Chem. 331, 198-202 (1996).

[3] S. Capone, A. Forleo, L. Francioso, R. Rella, P. Siciliano, J. Spadavecchia, and D. S. Presicce, "Solid state gas sensors: State of the art and future activities," J. Optoelecton. Adv. M. 5(5), 1335-1348 (2003).

[4] A. Honne, H. Odegard, H. Schumann-Olsen, H. Mosebach, D. Kampf, T. Stuffler, and G. Tan, "ANITA-Preparing for Automatic Air Analyses on the ISS," in Proceedings of International Conference On Environmental Systems (SAE - Society of Automotive Engineers, Rome, 2005).

[5] "OptoSense," http://www.optosense.com/.

[6] "GasSecure," http://www.gassecure.com/.

[7] D. A. Long, The Raman effect: a unified treatment of the theory of Raman scattering by molecules (John Wiley a Sons Ltd, West Sussex, 2002).

[8] S. Biedrzycki, Advanced techniques for gas-phase raman spectroscopy (Master thesis, University of Pittsburgh, 2011).

[9] J. Kiefer, T. Seeger, S. Steuer, S. Schorsch, M. C. Weikl, and A. Leipertz, "Design and characterization of a Raman-scatteringbased sensor system for temporally resolved gas analysis and its application in a gas turbine power plant," Meas. Sci. Technol. 19(8), 085408 (2008).

[10] J. B. Slater, J. M. Tedesco, R. C. Fairchild, and I. R. Lewis, "Raman spectrometry and its adaption to the industial environment," in Handbook of Raman Spectroscopy: From the Research Laboratory to the Process Line I. R. Lewis, and H. G. M. Edwards, eds. (Marcel Dekker Inc., New York City, 2001).

[11] M. E. Andersen, and R. Z. Muggli, "Microscopical techniques in the use of the molecular optics laser examiner Raman microprobe," Anal. Chem. 53(12), 1772-1777 (1981).

[12] "ThermoScientific - TruScan," http://www.ahurascientific.com/ material-verification/products/truscan/.
[13] "OceanOptics - IDRaman mini," http://oceanoptics.com/product/ idraman-mini/.

[14] “SciAps - Inspector500," http://sciaps.com/portable-raman-spectrometers/inspector-500/.

[15] M. A. Young, D. A. Stuart, 0. Lyandres, M. R. Glucksberg, and R. P. Van Duyne, "Surface-enhanced Raman spectroscopy with a laser pointer light source and miniature spectrometer," Can. J. Chem. 82(10), 1435-1441 (2004).

[16] Y. Oki, J. Takafuji, and M. Maeda, "Nonlinear Raman spectroscopies with Raman shifter for sensitive gas detection," IEEE LEOS Ann. Mtg. 99, 191-193 (1999).

[17] R. A. Hill and D. L. Hartley, "Focused, multiple-pass cell for Raman scattering.," Appl. Optics 13(1), 186-92 (1974).

[18] K. C. Utsav, Development of a multiple-pass Raman spectrometer for flame diagnostics (Dissertation thesis, University of Texas, 2013).

[19] J. M. Tedesco, and J. B. Slater, "Ellipsoidal raman signal amplifier," U. S. Patent US 2014/0036347 A1 (2014).

[20] R. A. Hill, A. J. Mulac, and C. E. Hackett, "Retroreflecting multipass cell for Raman scattering," Appl. Optics 16(7), 2004-2006 (1977).

[21] X. Li, Y. Xia, L. Zhan, and J. Huang, "Near-confocal cavityenhanced Raman spectroscopy for multitrace-gas detection," Opt. Lett. 33(18), 2143-2145 (2008).

[22] X. Yang, A. S. P. Chang, B. Chen, C. Gu, and T. C. Bond, "High sensitivity gas sensing by Raman spectroscopy in photonic crystal fiber," Sensor. Actuat. B-Chem. 176, 64-68 (2013).

[23] W. F. Pearman, J. C. Carter, S. M. Angel, and J. W.-J. Chan, "Multipass capillary cell for enhanced Raman measurements of gases," Appl. Spectrosc. 62(3), 285-9 (2008).

[24] S. Brunsgaard Hansen, R. W. Berg, and E. H. Stenby, "High-pressure measuring cell for Raman spectroscopic studies of natural gas," Appl. Spectrosc. 55(1), 55-60 (2001).

[25] S. Ohara, S. Yamaguchi, M. Endo, K. Nanri, and T. Fujioka, "Performance characteristics of power build-up cavity for Raman spectroscopic measurement," Opt. Rev. 10(5), 342-345 (2003).

[26] R. Salter, J. Chu, and M. Hippler, “Cavity-enhanced Raman spectroscopy with optical feedback cw diode lasers for gas phase analysis and spectroscopy," Analyst 137, 4669-4676 (2012).

[27] "Atmosphere Recovery Inc," http://www.atmrcv.com/technology. html.

[28] B. E. A. Saleh and M. C. Teich, Fundamentals of Photonics (John Wiley a Sons Ltd, New York, 1991).

[29] E. Voges, and K. Petermann, Optische Kommunikationstechnik (Springer, Heidelberg, 2002).

[30] J. D. Koch, and R. K. Hanson, "Temperature and excitation wavelength dependencies of 3-pentanone absorption and fluorescence for PLIF applications," Appl. Phys. B-Lasers 0. 76(3), 319-324 (2003).

[31] T. Bakke, A. Vogl, 0. Żero, F. Tyholdt, I.-R. Johansen, and D. Wang, "A novel ultra-planar, long-stroke and low-voltage piezoelectric micromirror," J. Micromech. Microeng. 20(6), 064010 (2010).

[32] T. Bakke, and I.-R. Johansen, "A robust, non-resonant piezoelectric micromirror," in Proceedings of 16th International Conference on Optical MEMS and Nanophotonics 171-172 (IEEE, Istanbul, 2011).

[33] E. Kikkinides, R. Yang, and S. Cho, "Concentration and recovery of carbon dioxide from flue gas by pressure swing adsorption," Ind. Eng. Chem. Res. 32, 2714-2720 (1993). 
[34] X. Xu, C. Song, R. Wincek, J. M. Andresen, B. G. Miller, and A. W. Scaroni, "Separation of $\mathrm{CO}_{2}$ from Power Plant Flue Gas Using a Novel $\mathrm{CO}_{2}$ 'Molecular Basket ' Adsorbent," Fuel Chem. Div. Prepr. 48(1), 162-163 (2003).

[35] S. C. Eichmann, J. Kiefer, J. Benz, T. Kempf, A. Leipertz, and T. Seeger, "Determination of gas composition in a biogas plant using a Raman-based sensor system," Meas. Sci. Technol. 25(7), 075503 (2014).
[36] B. Belaissaoui, G. Cabot, M.-S. Cabot, D. Willson, and E. Favre, "An energetic analysis of $\mathrm{CO}_{2}$ capture on a gas turbine combining flue gas recirculation and membrane separation," Energy 38(1), 167175 (2012). 